\title{
Bichromatically driven double well: parametric perspective of the strong-field control landscape reveals the influence of chaotic states
}

\author{
Astha Sethi and Srihari Keshavamurthy \\ Department of Chemistry, Indian Institute of Technology, Kanpur, India 208016
}

\begin{abstract}
The aim of this work is to understand the influence of chaotic states in control problems involving strong fields. Towards this end, we numerically construct and study the strong field control landscape of a bichromatically driven double well. A novel measure based on correlating the overlap intensities between Floquet states and an initial phase space coherent state with the parametric motion of the quasienergies is used to construct and interpret the landscape features. "Walls" of no control, robust under variations of the relative phase between the fields, are seen on the control landscape and associated with multilevel interactions involving chaotic Floquet states.
\end{abstract}

\section{INTRODUCTION}

Controlling quantum phenomena in atomic and molecular systems is an active area of research today. Persistent theoretical and experimental efforts have led to the formulation of several ingenious control schemes ${ }^{1,2,3,4}$ for both atomic and molecular systems. Recently researchers have been interested in determining the "control landscape" for specific systems $5,6,7,8,9,10,11,12,13$. The motivation for undertaking such extensive studies is atleast twofold. Firstly, several optimal control efforts $5,6,14$ seem to succeed despite employing local search algorithms in a prohibitively high dimensional control parameter space. Rabitz and coworkers ${ }^{5.6}$ reason that the topology of the control landscape can shed light on the robustness of the control strategies. Secondly, many control schemes 3 involve few, carefully chosen, levels which provide valuable insights into the control process. However, such few-level schemes might be compromised due to several reasons $15,16,17,18,19$ and one hopes that identifying the features of the control landscape associated with the deviations might lead to better control techniques.

In order to set the stage for our work we briefly discuss the concept of a control landscape. Consider a Hamiltonian of the form $H=H_{0}-\mu \mathcal{E}(t)$ with $\mu$ being the dipole operator and $\mathcal{E}(t)$ being the control field. There are two perspectives on the control problem which are of interest to the present work. The first one involves using a given field $\mathcal{E}(t ; \boldsymbol{\lambda})$ characterized by one or more control variables $\boldsymbol{\lambda}=\left(\lambda_{1}, \lambda_{2}, \ldots, \lambda_{f}\right)$. The objective in this case is to study the variation of, say, the transition probability $P_{i f} \equiv|\langle f|U(T, 0)| i\rangle|^{2}$ between an initial state $|i\rangle$ and a final state $|f\rangle$ at some target time $T$. The control landscape is constructed by viewing the transition probability as a functional of the field parameters i.e., $P_{i f}=P_{i f}[\mathcal{E}(t ; \boldsymbol{\lambda})]$. Regions on the landscape with high values for $P_{\text {if }}$ yield the best possible choice of the control variables in the presupposed $\mathcal{E}(t ; \boldsymbol{\lambda})$. Note that it is quite possible to have several distinct choices for the control variables which yield the same value for $P_{i f}$. Several such examples of control landscapes can be found in the literature $2,7,8,9,10,13$

The second perspective on the control problem comes from the optimal control theory (OCT) approach 4,6 . In
OCT a cost (landscape) function of the form $\Phi(U)=$ $\operatorname{Tr}\left[U \rho(0) U^{\dagger} \Theta\right] \equiv\langle\Theta\rangle$, with $\rho(0)$ being the initial density matrix and an arbitrary observable $\Theta$, is extremized with respect to the field $\mathcal{E}(t)$. The optimal fields correspond to the solutions of the variational condition $\delta \Phi(U) / \delta \mathcal{E}(t)=0$ wherein the unitary time evolution operator $U=U[\mathcal{E}(t)]$ is naturally considered as a functional of the field. The transition probability $P_{i f}$ is a special case of $\Phi(U)$ with $\rho=|i\rangle\langle i|$ and $\Theta=|f\rangle\langle f|$. Again, the set of optimal fields that yield the same $\langle\Theta\rangle$ constitutes a control level set whose topology is of considerable interest since one can imagine 'morphing' an experimentally undesirable optimal field to a more desirable field. In order to be able to carry out such a morphing it is essential to have full control of the system. Assuming full controllability Rabitz and coworkers have explored the topology of the OCT landscapes in great detail ${ }^{6}$. A particularly striking result is that the full quantum control landscape is devoid of false traps or suboptimal extrema ${ }^{5,6}$. However, in practice one is invariably forced to impose constraints on $\Phi(U)$ in various forms. Examples include specification of target time $T$, minimizing the field fluence, and reduced or restricted parametrizations of the control field.

Among the two perspectives on control outlined above we adopt the first one in this work i.e., the nature of the control landscape with a given form of the field. Although we do not undertake an OCT calculation in the present work, it is nevertheless useful to think of the field used herein as a result of a reduced dimensional parametrization of an optimal field coming out of a OCT calculation. The reason being that restricted parametrizations of the optimal $\mathcal{E}(t)$ typically projects the full OCT control space to a lower dimensional space which can exhibit suboptimal features 6 . 'Suboptimal' features on the control landscape are, therefore, expected in our analysis. Clearly, in the OCT context, suboptimal features can be avoided if the restricted parametrization is done carefully by preserving the overall intrinsic topology of the control landscape and level sets. Discovering such appropriate parametrizations might not be easy but a recent combined experimental and theoretical work by Roslund et al. provides a clear example ${ }^{20}$. In certain instances, as in the work by Bartelt et al., a systematic 
study of simple parametrizations of the optimal field can provide mechanistic insights into the control process 21 .

Based on the discussion above, one can interpret the suboptimal features as artificial due to inappropriate field parametrization or as genuine due to the unavoidable constraints imposed on the system. Consequently, the model study presented in this work incorporates two of the constraints that are expected to result in a complicated control landscape - strong fields and simple forms of the control field. It has been suggested that in strong field regimes local traps may appear more readily. However, even in the strong field regimes recent studies indicate that interpreting the various features of the constrained field control landscape is far from being straightforward $10,11,12$. In this context, it is well known that the classical phase space of strongly driven systems exhibits widespread chaos. Could it be that local traps on the strong field control landscapes arise due to the chaos? Unfortunately, not much is known about the impact of chaotic or delocalized quantum states on the topology of control landscapes and level sets. Such an analysis for the full OCT landscape is far from being simple. Therefore, in this work we make a beginning, within the perspective discussed above, to understand the nature and origins of the suboptimal features from a classical-quantum correspondence viewpoint.

In most physical systems the phase space is mixed regular-chaotic and there is sufficient evidence in the literature to indicate that quantum states delocalized in the chaotic regions can significantly influence the control of initial states localized in the regular regions. For instance, $\mathrm{Na}$ and Reichl ${ }^{19}$ have shown that adiabatic passage mechanisms are modified due to the presence of chaotic states leading to chaos-assisted passage. Gong and Brumer $\underline{18}$ have recently reviewed the subject of coherent control of quantum phenomenon in the presence of chaos. Their studies, especially using the (modified) kicked rotor, have highlighted several intriguing features like faster-than-classical quantum anomalous diffusion and the nontrivial influence of classical phase space structures with area much smaller than the effective Planck constant. As a last example we mention the work by Takami and Fujisaki22 on optimal control of random matrix systems. Random matrix systems correspond to strongly chaotic classical phase space with no regular regions. In such cases it is possible, using OCT, to obtain an analytic expression for the optimal field which controls the transition between two random states. The aforementioned studies, and other earlier investigations, concerned with the control of quantum processes in classically chaotic systems have raised many interesting issues. However, there are very few attempts to analyze the various features on the control landscape in terms of the phase space nature of the quantum states. Controllability of quantum dynamics despite the strong chaos is surprising and calls for a careful study of the control landscape from a classical-quantum correspondence perspective.
We begin in section @ with a brief description of the model system, driven double well, which has served as a paradigm for many coherent control studies. The controllability of the dynamics using a bichromatic field is studied as a function of field parameters. The role of chaotic (delocalized) Floquet states in attempting to control the decay of an initial state localized in the regular region of the phase space is highlighted. Section [III introduces a measure which involves correlating the response of the Floquet states to the control field with the overlap intensities between the initial state and the Floquet states. We show that the correlator is related to generic landscape functionals and can therefore yield useful insights into the features of the control landscape from a classicalquantum correspondence point of view. In section IV we show that the proposed correlator is very sensitive to the underlying phase space structure and clearly identifies regions of no control involving delocalized (chaotic) Floquet states. Section $\nabla$ concludes with comments on the role of the chaotic states in the process of control and future studies.

\section{MODEL SYSTEM}

The model of choice to illustrate our approach is the driven double well system. The Hamiltonian is given by

$$
H(x, p ; t)=\frac{1}{2 m} p^{2}+B x^{4}-D x^{2}+x \mathcal{E}(t)
$$

with $\mathcal{E}(t)$ being the field. The driven double well model is an ideal testbed for several schemes that have been suggested for controlling coherent superposition of quantum states $2,13,23,24,25,26$. Apart from being used to elucidate phenomena like chaos-assisted tunneling 27 and coherent destruction of tunneling 24 , the model above is also relevant in research areas like quantum computing 28 and quantum dots 29 . In this context we also note an early study ${ }^{30}$ by Schwieters and Rabitz on the above system with the aim of designing an optimal $\mathcal{E}(t)$ to reduce the difference between quantum observables and their classical analogs. In the present work, as alluded to in the introduction, our goal is to study control using a presupposed field and the resulting influence on the control landscape. A variety of choices can be made for $\mathcal{E}(t)$ and in this work we choose a bichromatic form

$$
\mathcal{E}(t)=\lambda_{1} \cos (\omega t)+\lambda_{2} \cos (2 \omega t+\phi)
$$

The above choice for $\mathcal{E}(t)$ is motivated by a large number of studies ${ }^{31}$ that have shown the utility of such fields in controlling the dynamics with the possibility of using the relative phase $\phi$ as an extra control knob. Moreover, due to the periodicity of Eq. 1. $H(x, p, t)=H\left(x, p, t+T_{f}\right)$, with period $T_{f}=2 \pi / \omega$, the dynamics can be conveniently analyzed ${ }^{33}$ using Floquet theory ${ }^{34.35}$ in terms of the Floquet states $\left\{\left|\chi_{n}\right\rangle\right\}$ and the associated quasienergies $\left\{E_{n}\right\}$. 
Throughout this work $\hbar=1$ and we fix the parameter values $^{26,27} m=1, D=10, B=0.5$ and, $\omega=6.07$. The double well supports about eight tunneling doublets and $\omega$ nearly corresponds to the energy separation between the ground and first excited states, neglecting the tunnel splittings. The primary field strength $\lambda_{1} \in[6.0,11.0]$ is chosen such that the classical phase space, for $\lambda_{2}=0$, has two symmetry related regular islands embedded in a chaotic sea (cf. inset in Fig. 1A). The symmetry related regular regions and the associated symmetric pairs of Floquet states exist due to the discrete symmetry $H(p, x, t)=H(-p,-x, t+\pi / \omega)$. The parameter values have been chosen to make contact with an earlier work by Farrelly and Milligan ${ }^{26}$ which explored the possibility of controlling the tunneling using bichromatic fields. Different choices for the parameters, as long as the phase space exhibits substantial chaos, show similar behaviour and do not alter our conclusions.

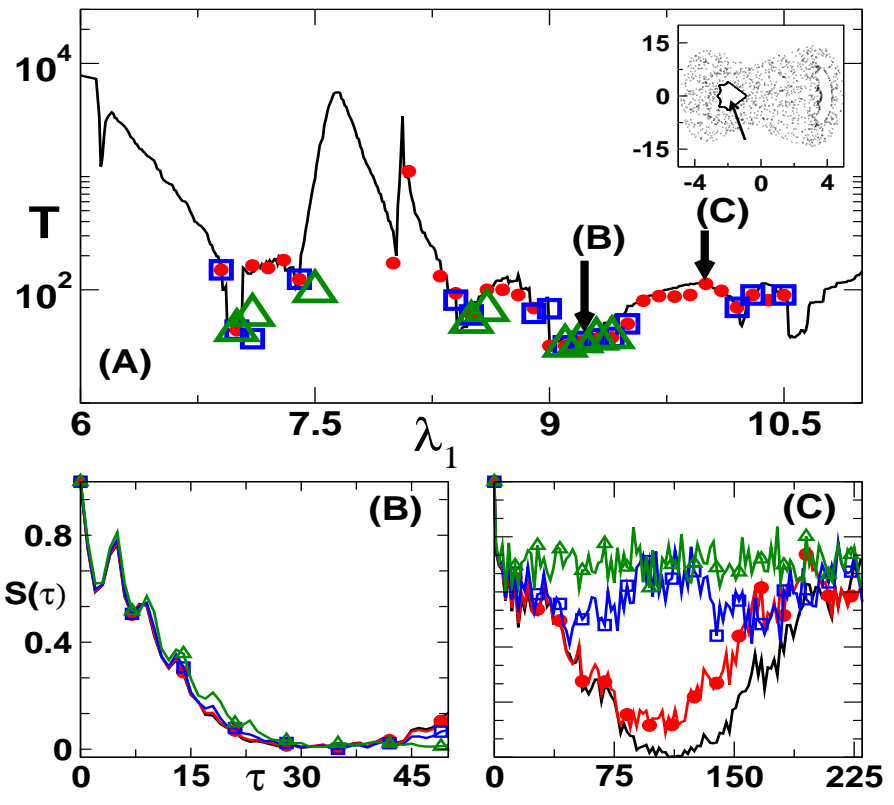

FIG. 1: (Color online) (A) Decay time $T$ versus the field strength $\lambda_{1}\left(\lambda_{2}=0\right)$ for the coherent state, $|z\rangle \equiv\left|x_{0}, p_{0}\right\rangle=$ $|-1.5,0\rangle$, localized in the left regular island (indicated by an arrow in the inset showing the stroboscopic phase space at $\left.\lambda_{1}=6.4\right)$. (B) and (C) show the influence of the $2 \omega$-control field with $\phi=0$ on the survival probabilities for $\lambda_{1}=9.2$ and 10 respectively. Increasing $\lambda_{2}$ from 0 (black) to 0.2 (red circles), 0.7 (blue squares), and 2.1 (green triangles) leads to localization in case (C) but not in case (B). The same information, with consistent color code, is shown in (A) as points unaffected by increasing $\lambda_{2}$.

\section{A. Control landscape: influence of the chaotic states}

The control problem that we address is as follows. The initial state, as in the earlier studies ${ }^{26,27}$, is a coherent state $|z\rangle \equiv\left|x_{0}, p_{0}\right\rangle$ localized in the phase space about $\left(x_{0}, p_{0}\right)$ within one of the regular islands. The coherent state is constructed as linear superposition

$$
\left|x_{0}, p_{0}\right\rangle=\sum_{b=0}^{\infty} c_{b}\left(x_{0}, p_{0}\right)|b\rangle
$$

of a harmonic oscillator basis $\{|b\rangle\}$ with frequency $\omega_{b}=$ 6.25 (corresponding to $\left\langle(\Delta x)^{2}\right\rangle=0.08$ ). The coefficients are given by

$$
\begin{aligned}
c_{b}\left(x_{0}, p_{0}\right) & =\frac{1}{\sqrt{b !}} \alpha^{b} e^{-|\alpha|^{2} / 2} e^{-i x_{0} p_{0} / 2} \\
\alpha & =\sqrt{\frac{\omega_{b}}{2}}\left(x_{0}+\frac{i}{\omega_{b}} p_{0}\right)
\end{aligned}
$$

We employ the same basis to compute the Floquet states and quasienergies used in the rest of this work. The time evolved state $|z(t)\rangle$ can be expressed in terms of the Floquet states as

$$
|z(t)\rangle=\sum_{n} e^{-i E_{n} t}\left|\chi_{n}(t)\right\rangle\left\langle\chi_{n}(0) \mid z(0)\right\rangle
$$

Measuring time in units of $T_{f}$ and owing to the periodicity of the Floquet states, $\left|\chi_{n}(t)\right\rangle=\left|\chi_{n}\left(t+T_{f}\right)\right\rangle$, the above equation simplifies to

$$
|z(\tau)\rangle=\sum_{n} e^{-i E_{n} \tau}\left|\chi_{n}(0)\right\rangle\left\langle\chi_{n}(0) \mid z(0)\right\rangle
$$

with $\tau \equiv k T_{f}$ and integer $k$. In the absence of the $2 \omega$-field ("control field") $|z\rangle$ decays over a timescale $T$ which is determined from the first vanishing of the survival probability

$$
\begin{aligned}
S(\tau) & \equiv|\langle z(0) \mid z(\tau)\rangle|^{2} \\
& =\left|\sum_{n} e^{-i E_{n} \tau}\left\langle z(0) \mid \chi_{n}(0)\right\rangle\left\langle\chi_{n}(0) \mid z(0)\right\rangle\right|^{2} \\
& =\sum_{m, n} p_{z n} p_{z m} e^{-i\left(E_{n}-E_{m}\right) \tau}
\end{aligned}
$$

where the overlap intensities are denoted by $p_{z n} \equiv$ $\left|\left\langle z(0) \mid \chi_{n}(0)\right\rangle\right|^{2}$. Note that $S(\tau)$ is just a special case of $P_{i f}$ with $|i\rangle=|f\rangle=|z\rangle$. In Fig. 11A the decay time is shown as a function of $\lambda_{1}$ for $\lambda_{2}=0$. The strong fluctuations in $T$, despite similar phase space nature over the entire range of $\lambda_{1}$, is a direct consequence of the underlying $\operatorname{chaos}^{27}$. Recently it has been established ${ }^{36,37}$ that the local structure of the phase space about the location of $|z\rangle$ can play a crucial role in the decay dynamics. The local phase space structure around the left regular island is shown in Fig. 2 for various values of $\lambda_{1}$. Comparing the features of the decay time plot in Fig. 11A with the local phase space nature in Fig. 2 shows qualitative correspondence. In particular, the drastic variation of $T$ around $\lambda_{1}=8$, seen in Fig. 17A, is reflected in the significant change of the local phase space nature in Fig. 2(b) 

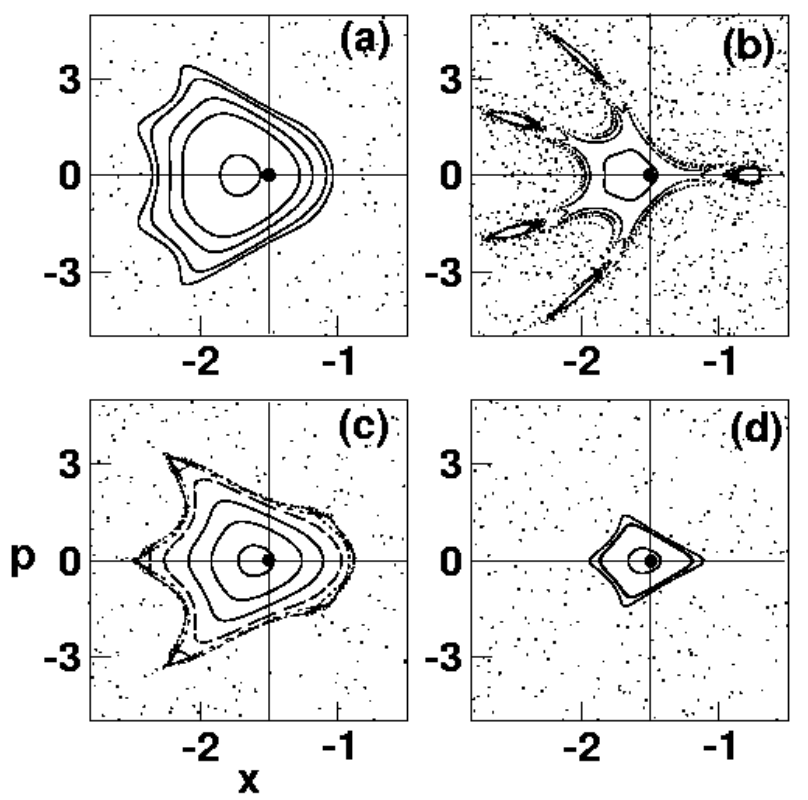

FIG. 2: The local nature of the phase space in the vicinity of the initial state $|z\rangle$ (black circle) for $\lambda_{1}$ equal to (a) 7.0 (b) 8.0 (c) 9.0 and (d) 10.0 with $\lambda_{2}=0$. Note the drastic change in (b) which is mirrored in the decay time behavior in Fig. 11A.

as compared to those in Fig. 2(a),(c), and (d). There is also preliminary evidence (not discussed in this work) which indicates that the various field-matter nonlinear resonances control the decay of $|z\rangle$ and partly responsible for some of the structure seen in Fig. 11A.

Is it possible to control the decay of $|z\rangle$ with an appropriate choice of the parameters $\left(\lambda_{2}, \phi\right)$ of the $2 \omega$-field? In this instance the problem of control, in the asymptotic limit $\tau \rightarrow \infty$, is equivalent to the suppression of tunneling between the two symmetry related islands in the phase space. At the outset one expects, for $\lambda_{2} \neq 0$ and $\phi \neq \pi / 2$, the discrete symmetry to be broken leading to localization and hence perfect control. However, the results of the computations summarized in Fig. 1] show a more complicated behaviour. For most of this study we will choose two representative cases to illustrate the issues. In the first case, corresponding to $\lambda_{1}=10.0$ and labeled as (C) in Fig. 1 a monotonic increase of $\lambda_{2}$ with $\phi=0$ results in the localization of $|z\rangle$. This is in accordance with the earlier observations by Farrelly and Milligan $^{26}$. On the other hand, the decay dynamics for the second case, corresponding to $\lambda_{1}=9.2$ and labeled as (B) in Fig. 1. is unaffected by the control field even for fairly large values of $\lambda_{2}$.

To obtain a global picture, in Fig. 3 the control landscape for $\phi=0$ is shown in terms of the time-smoothed survival probability

$$
\langle S\rangle=\frac{1}{\tau} \int_{0}^{\tau} d \tau^{\prime} S\left(\tau^{\prime}\right)
$$

as a function of the field parameters $\left(\lambda_{1}, \lambda_{2}\right)$. Note that the choice of $\langle S\rangle$ to represent the landscape is made for convenience; the decay time is a better choice which requires considerable effort but the gross qualitative features of the control landscape do not change upon using $\langle S\rangle$. Large (small) values of $\langle S\rangle$ indicate that the decay dynamics is suppressed (enhanced). It is clear from Fig. 3 that the landscape is highly convoluted - a consequence of the simple bichromatic choice for $\mathcal{E}(t)$ and the nonlinear nature of the classical dynamics. An earlier OCT study ${ }^{32}$ on a different classically chaotic system has also suggested that the control landscape could be highly convoluted. From a control point of view, there are regions on the landscape for which a monotonic increase of $\lambda_{2}$ leads to increasing control. At the same time there are regions, especially around $\lambda_{1} \approx 9.2$, where increasing $\lambda_{2}$ hardly effects the decay dynamics and indicates very little control. As noted before, one expects such features even on constrained OCT landscapes. In this work we would like to understand the nature and origin of such robust regions of no control.

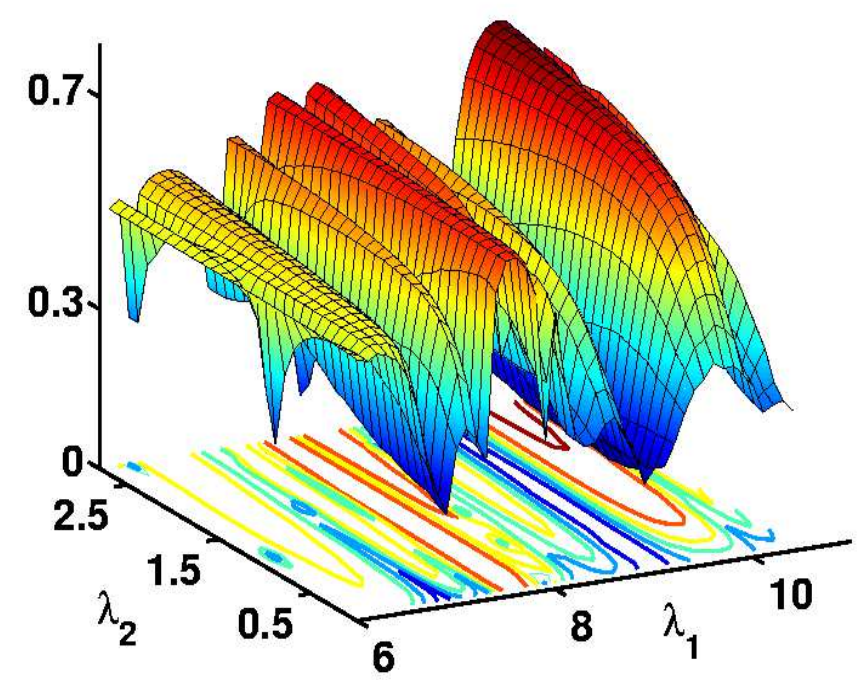

FIG. 3: (Color online) Time smoothed survival probability representation of the control landscape for $\phi=0$. The initial state is the same as in Fig. 1 Notice the convoluted form of the landscape with the regions of low probability indicating little to no control.

Insights into the lack of control can be obtained by studying the variation of the Floquet quasienergies with $\lambda_{2}$. In Fig. $4 \mathrm{~A}$ we show the results of such a computation corresponding to region B (cf. Fig. 1). Over the entire range of $\lambda_{2}$ six states contribute to the decay of $|z\rangle$ as evident from the overlap intensities shown in Fig. 4B. A closer look reveals the existence of states delocalized in the chaotic regions of the phase space two such states are shown in Fig. $4 \mathrm{~B}$ in terms of their Husimi distributions 27 . In contrast, Fig. $4 \mathrm{C}$ shows that the dynamics of case (C) in Fig. 1 is dictated by localized states which explains the ease of control with the 

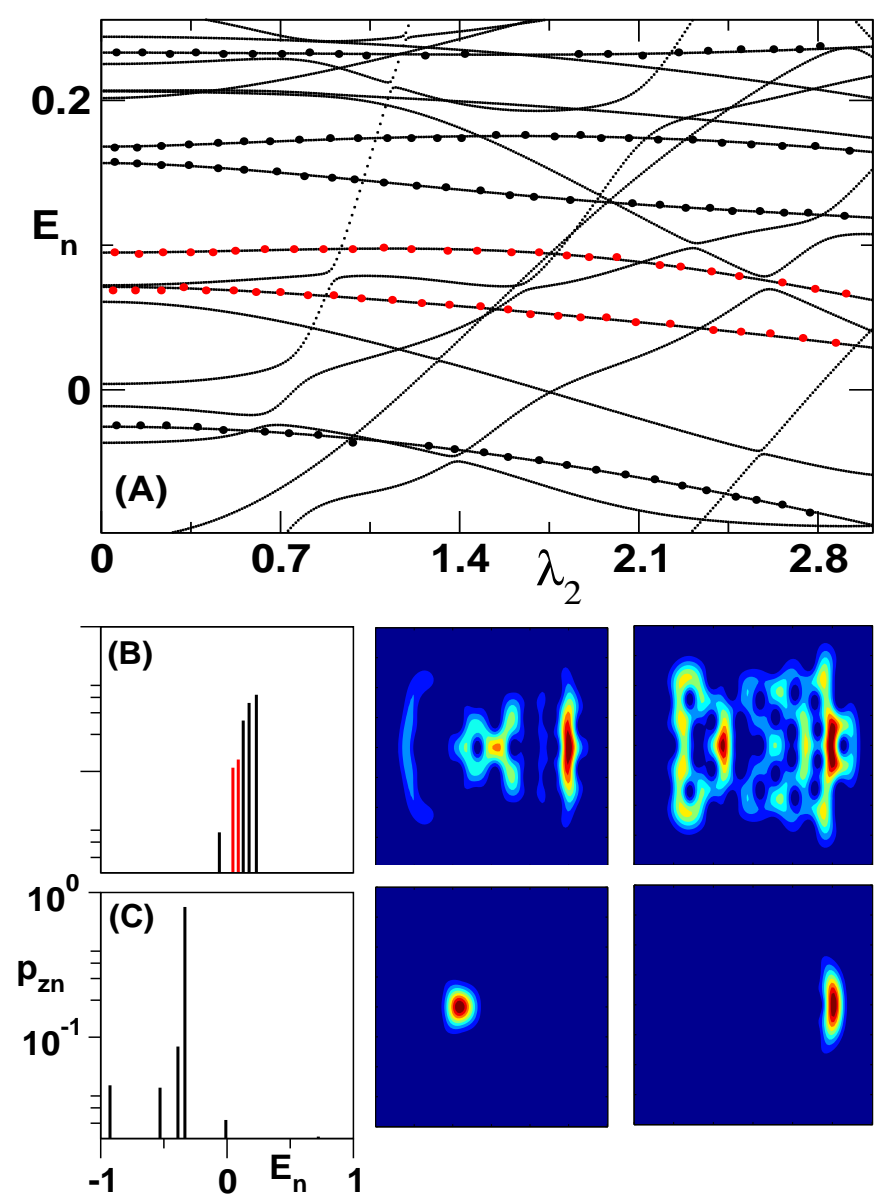

FIG. 4: (Color online) (A) Variation of the Floquet quasienergies with $\lambda_{2}$ and fixed $\lambda_{1}=9.2$. Six states that have appreciable overlap with $|z\rangle$ are highlighted by circles. (B) Overlap intensity $p_{z n}$ for $\lambda_{1}=9.2$ and $\lambda_{2}=2.1$ indicates multilevel interactions involving the states shown in (A). Husimi distribution function of two delocalized Floquet states (red) are also shown. (C) Same as in (B) with $\lambda_{1}=10$ and $\lambda_{2}=0.7$ shows the localized nature of the participating states.

symmetry breaking $2 \omega$-field. Therefore it is reasonable to expect that the lack of control, signalled by plateaus in Fig. 1A and the valleys in Fig. 3, is due to the dominant participation by chaotic states i.e., chaos-assisted tunneling 38 . The plateaus arise due to the fact that the coupling between the localized states and the delocalized states vary very little with increasing control field strength $\lambda_{2}$ - something that is evident from the Floquet level motions shown in Fig. 44 A and established earlier by Tomsovic and Ullmo in their seminal work ${ }^{38}$ on chaosassisted tunneling in coupled quartic oscillators. It is important to note that for $\lambda_{2}=0$ the chaotic states, as opposed to the regular states, do not have a definite parity. Consequently, presence of the $2 \omega$-field does not have a major influence on the chaotic states $\frac{17}{}$. Thus, if one or more chaotic states are already influencing the dynamics of $|z\rangle$ at $\lambda_{2}=0$ then the bichromatic control is expected to be difficult.
Similar conclusions were arrived at in a previous study 17 involving bichromatically driven pendulum. However, several questions, not addressed by the previous studies, arise at this juncture. What is the precise role of the chaotic states for control? Are the plateaus robust for varying $\lambda_{2}$ and $\phi$ ? Is there a suitable measure, generalizable to systems with higher degrees of freedom, that can identify regions on the control landscape with little or no control?

\section{SENSITIVE MEASURE FOR THE CONTROL LANDSCAPE}

In order to address the questions raised above we first suggest a measure, different from $S(\tau)$ or $\langle S\rangle$, to map out the control landscape. Note that the Floquet states and quasienergies of Eq. 1 are parametrically dependent on the field strengths i.e., $E_{n}=E_{n}(\boldsymbol{\lambda})$ and $\left|\chi_{n}\right\rangle=\left|\chi_{n}(\boldsymbol{\lambda})\right\rangle$. Thus the response of a Floquet state to the $2 \omega$-field, as shown in Fig. 44 A, is measured by the parametric derivative $\partial E_{n} / \partial \lambda_{2}$ which is also called as the level "velocity". At the same time the decay dynamics of a coherent state $|z\rangle$ is dominated by Floquet states that have appreciable overlap with $|z\rangle$ (cf. Eq. 6). Therefore, Floquet states $\left|\chi_{n}\right\rangle$ with substantial overlap $p_{z n}$ and finite $\partial E_{n} / \partial \lambda_{2}$ are expected to be important in controlling the dynamics of $|z\rangle$. The qualitative argument can be made quantitative by introducing the overlap intensity-level velocity correlator

$$
C_{z}\left(\lambda_{1}, \lambda_{2}\right)=\frac{1}{\sigma_{z} \sigma_{E}}\left\langle p_{z n} \frac{\partial E_{n}(\boldsymbol{\lambda})}{\partial \lambda_{2}}\right\rangle_{n}
$$

$C_{z}(\boldsymbol{\lambda})$ was first proposed by Tomsovic ${ }^{39}$ as a sensitive measure for deviations from ergodicity in strongly chaotic systems and has been studied in detail in subsequent works $\underline{40}$. In Eq. 8, $\sigma_{z}^{2}$ and $\sigma_{E}^{2}$ are the local variances of $p_{z n}$ and $\partial E_{n} / \partial \lambda_{2}$ respectively and the average is over all the Floquet states.

The sensitivity of the correlator to phase space structures has been amply demonstrated in the previous studies $\underline{40}$. Since the earlier works did not explicitly deal with driven systems, in Fig. 5 we give one example of the sensitivity in the present context. In Fig. 5 we place coherent states at different phase space points on the phase space and obtain a correlator map. Note that the coherent state parameters are identical to the ones used in the earlier sections. The correlator map is shown for two different field strengths (A) $\left(\lambda_{1}, \lambda_{2}\right)=(9.2,0.7)$ and (B) $(10.0,0.7)$ with $\phi=0$. We remind the reader that these parameter values correspond to the two exemplary cases denoted as (B) and (C) in Fig. 1(A). Several observations can be made by inspecting the figure and we mention a few important ones. Firstly, the inhomogeneous nature of the phase spaces is reflected by the correlator. Specifically, the large values of $C_{z}$ in the regular islands on the periphery of the phase space imply participation of "fast moving" Floquet states in the dynamics. Examples of 

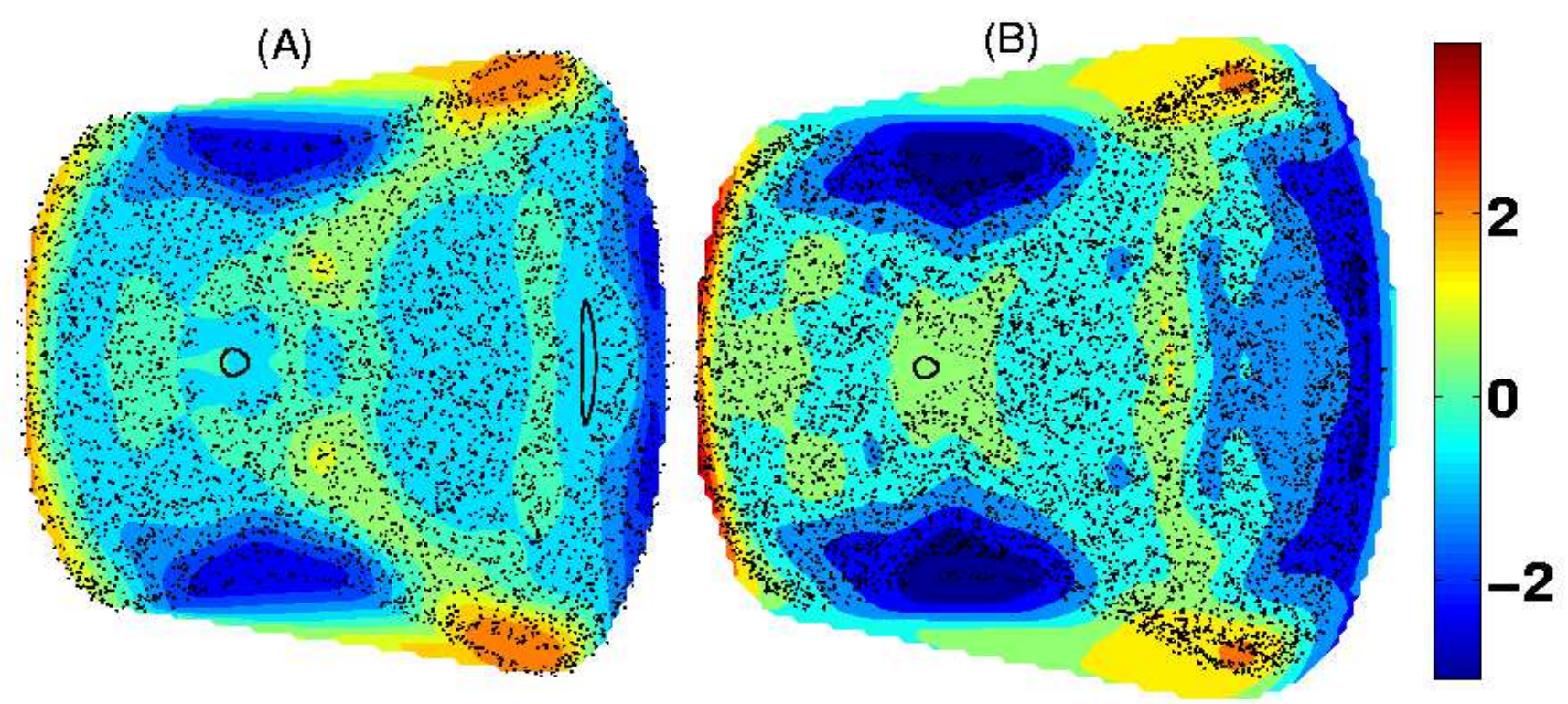

FIG. 5: (Color online) The intensity-velocity correlator (cf. Eq. 8) computed over the entire phase space at $\left(\lambda_{2}=0.7, \phi=0\right)$ for (A) $\lambda_{1}=9.2$ (B) $\lambda_{1}=10.0$. Calculations are performed by placing coherent states at the different phase space points. Note the similarity of the phase space but the essential differences in the correlator maps. See text for details.

such Floquet states can be seen in Fig. 4(A) as states with large level velocities. Secondly, the correlator takes on different values even in the chaotic regions and therefore points to the different nature of the dynamics i.e., different groups of Floquet states that influence the dynamics. Finally, despite the similar looking phase spaces, it is clear from Fig. [5 that the correlator maps are different. In particular, note that the correlator values in Fig. $5 \mathrm{~A}$ about the left and the symmetry related right regular islands have the same sign. However, in Fig. 5B the same regions have correlators of opposite signs. This indicates a break in symmetry and hence provides some hints to the fact that one obtains control in this case as opposed to in case A.

Before proceeding further we note that the correlator is related to the generic landscape functionals employed in OCT studies ${ }^{6}$. In order to see this consider the generic quantum control landscape functiona $\underline{1}^{\underline{6}}$

$$
\Phi(U)=\operatorname{Tr}\left[U(\tau) \rho(0) U(\tau)^{\dagger} \Theta\right]
$$

with $\rho(0), \Theta$, and $\tau$ being the initial density matrix, an arbitrary observable operator, and final dynamical time respectively. Choosing $\rho \equiv|z\rangle\langle z|$ and $\Theta \equiv \mathcal{E}_{2 \omega}(x, t) \equiv$ $x \cos (2 \omega t+\phi)$ one can express the landscape functional as

$$
\Phi(U)=\sum_{n}\left\langle\chi_{n}(0) \mid z(\tau)\right\rangle\left\langle z(\tau)\left|\mathcal{E}_{2 \omega}\right| \chi_{n}(0)\right\rangle
$$

Using Eq. 5 one can write

$$
\begin{aligned}
\Phi(U) & =\sum_{m, n} e^{-i\left(E_{n}-E_{m}\right) \tau}\left\langle\chi_{n} \mid z\right\rangle\left\langle z \mid \chi_{m}\right\rangle\left\langle\chi_{m}\left|\mathcal{E}_{2 \omega}(\tau)\right| \chi_{n}\right\rangle \\
& =\sum_{n} p_{z n}\left(\frac{\partial E_{n}(\boldsymbol{\lambda})}{\partial \lambda_{2}}\right)+\text { nondiagonal terms }
\end{aligned}
$$

The first term on the last line in Eq. 11 comes about due to the Hellman-Feynman theorem. The diagonal term in Eq. 11 is essentially the correlator in Eq. 8 and the contribution from the nondiagonal terms can be neglected in the long time limit. The variances in Eq. 8 ensure that $C_{z}$ is a true correlation function. However, without the variances one is dealing with the covariance which is related to the landscape functional $\Phi$ via Eq. 11. Note that the covariance already has the essential features that are needed for our study and the existing semiclassical insights $\frac{40}{*}$ should prove valuable in the context of the present work.

What should one expect from the correlator? In general, $C_{z}(\boldsymbol{\lambda})$ highlights the presence of groups of states exhibiting common localization features 39,40 . In the ergodic limit it is known 39 that $C_{z}(\boldsymbol{\lambda}) \sim 0 \pm N^{-1 / 2}$ for any initial state $|z\rangle$ with $N$ being the number of states involved in the averaging in Eq. 8. However, despite the strong fields and extensive chaos, the system considered herein is not ergodic (cf. Fig. (5)). The interpretation is subtle as compared to the earlier studies $\underline{39,40}$ since chaotic states already exist for $\lambda_{2}=0$. The chaotic states, due to their delocalized nature, have small level-velocities $\left|\partial E_{n} / \partial \lambda_{2}\right|$ 

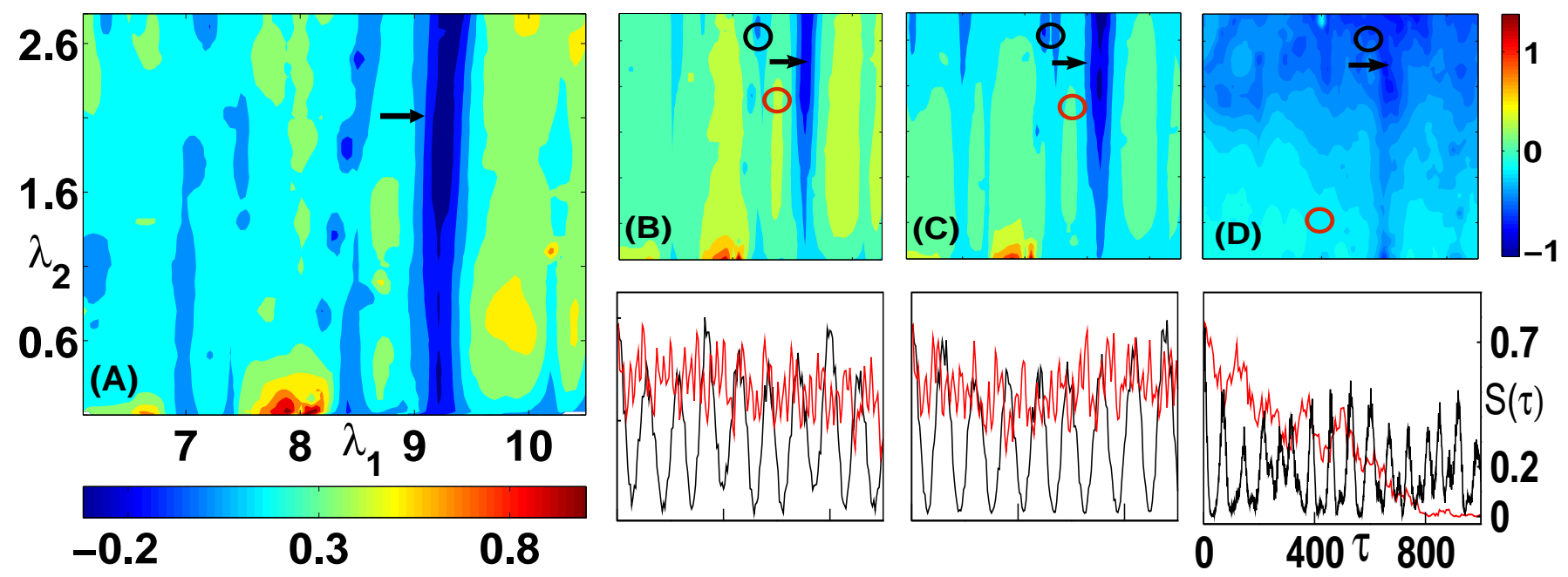

FIG. 6: (Color online) Control landscapes constructed via the intensity-level velocity correlator $C_{z}(\boldsymbol{\lambda})$ for phase $\phi$ equal to (A) 0 (B) $\pi / 4$ (C) $\pi / 3$ and (D) $\pi / 2$. All landscapes shown have the same axes range. Regions lacking control are associated with $C_{z}(\boldsymbol{\lambda}) \lesssim 0$. Note the one-to-one correspondence between the landscape in (A) and the one shown in Fig. 3 and the robustness of the "wall" of no control around $\lambda_{1} \approx 9.2$ (indicated by arrows). Survival probabilities shown in the lower panels for specific field parameters (indicated by circles) confirm the predictions based on $C_{z}(\boldsymbol{\lambda})$. Calculations of the landscape are performed on a $50 \times 30$ grid with a spacing of 0.1 .

which is evident from Fig. 4A as well. Consequently, participation of several chaotic states in the decay dynamics of $|z\rangle$ results in small value for $\left|C_{z}(\boldsymbol{\lambda})\right|$. As a simple model, assume $N_{c}$ chaotic states with nearly the same level velocities $v_{c}$ contribute equally to the dynamics of $|z\rangle$ i.e., similar intensities $p_{z c}=N_{c}^{-1}$. Note that the division of the total number of states as $N=N_{c}+N_{r}$, with $N_{r}$ being the number of regular states, is strictly valid only in the semiclassical limit. Clearly the parameters in this work are such that the system is far from the required limit but the arguments are presented with an attempt to understand some of the features of the correlator. Within the simple model, using the sum rule that the average of the level-velocities over all $N$ states is zero, it is possible to show that

$$
\begin{aligned}
\langle v\rangle_{r} & \equiv \frac{1}{N_{r}} \sum_{j=1}^{N_{r}} v_{j}=-v_{c} f_{\mathrm{ch}} \\
C_{z} & =v_{c}\left[\frac{f_{\mathrm{ch}}\left(1+f_{\mathrm{ch}}\right)}{v_{c}^{2} f_{\mathrm{ch}}+\left\langle v^{2}\right\rangle_{r}}\right]^{1 / 2} \approx-\frac{\langle v\rangle_{r}}{\left\langle v^{2}\right\rangle_{r}^{1 / 2}}
\end{aligned}
$$

with $f_{\text {ch }} \equiv N_{c} / N_{r}$ and the limiting form of the correlator above arises when $v_{c}^{-2}\left\langle v^{2}\right\rangle_{r} \gg f_{\text {ch }} \gg 1$. It is crucial to observe that the sign of the correlator in Eq. 13 is determined by the chaotic level-velocity $v_{c}$. An a priori knowledge of the sign of $v_{c}$ in a specific system is difficult but can be determined from a single Floquet calculation. Eq. 13 suggests that $\left|C_{z}\right|$ will be small for $\langle v\rangle_{r} \ll\left\langle v^{2}\right\rangle_{r}^{1 / 2}$.

\section{INTERPRETATION OF THE FEATURES ON THE CONTROL LANDSCAPE}

Armed with the qualitative insight into the behavior of $C_{z}(\boldsymbol{\lambda})$ we now compute the control landscape using Eq. 8 for the entire range of $\lambda_{1}$ shown in Fig. 11A. The resulting control landscape is shown in Fig. 6A for relative phase $\phi=0$ and should be compared to the landscape in Fig. 3 mapped via the time-smoothed survival probability $\langle S\rangle$. It is important to note that the two landscapes show identical features. In particular, the regions lacking control on the landscape in Fig. 3] correspond to the regions showing $C_{z}(\boldsymbol{\lambda}) \lesssim 0$ in Fig. 6A. However, using the correlator based landscape it is now possible to provide an explanation, not readily forthcoming from the landscape in Fig. 3, for the source of such lack of control. For the specific parameters chosen in this study the chaotic level-velocity distribution is peaked around a negative value and thus $C_{z}(\boldsymbol{\lambda}) \lesssim 0$, following the results of the simple model in Eq. 13 implies that the regions of no control arise due to the participation of chaotic Floquet states. Although the role of chaotic states to control is already highlighted in Fig. 团at a single point on the control landscape, it is worth mentioning that the correlator identifies such regions on the landscape with remarkable ease.

A noteworthy feature on both the control landscapes is the existence of "walls" of no control. Interestingly, the wall at $\lambda_{1} \approx 9.2$ is particularly robust and, hence, consistent with the results in Fig. 1 and Fig. 4 which suggest extensive participation by delocalized Floquet states. Therefore our observations establish $C_{z}(\boldsymbol{\lambda})$ to be a highly 
sensitive measure capable of identifying the lack of control due to the involvement of chaotic states. Several other regions of no control can be seen in Fig. 6 $6 \mathrm{~A}$ which are less robust in comparison to the one at $\lambda_{1}=9.2$. For instance, the walls around $\lambda_{1} \approx 7.0$, and 8.2 seem to break up at certain values of $\lambda_{2}$ but, interestingly, reappear at higher $\lambda_{2}$. In every instance where $C_{z}(\boldsymbol{\lambda}) \lesssim 0$ we have confirmed the presence of atleast one chaotic state with substantial $p_{z n}$. This points to a fairly nontrivial role played by the chaotic states in the control process. The walls and islands of no control result in the highly convoluted topology of the control landscape. Hence, using the symmetry breaking property of the $2 \omega$-field for control purposes is not very effective when chaotic Floquet states are participating in the dynamics. On the other hand regions with $C_{z}(\boldsymbol{\lambda})>0$ in Fig. 6A Andicate varying degree of control. Interestingly, an earlier analysis 26 , corresponding to case (C) in Fig. 1, happens to be in a region away from the walls of no control! Again, the large positive value of the correlator indicates the absence of interactions with chaotic states leading to localization due to symmetry breaking as seen in Fig. 4C.

Is it possible to break the wall of no control with a judicious choice of $\phi$ ? In order to explore such a possibility we show the control landscape for different choices of $\phi$ in Fig. 6 6 B, C, and D. The case $\phi=\pi / 2$ is shown in Fig. 6 $\mathrm{D}$ and one observes that $C_{z}(\boldsymbol{\lambda}) \lesssim 0$ over most of the landscape which indicates very little control. This is consistent with the fact that the combined driving field is symmetric about the time axis for $\phi=\pi / 2$. The control landscape for $\phi=\pi / 3$, and $\pi / 4$ are also shown in Fig. $6 \mathrm{C}$ and $\mathrm{B}$ respectively. Note that decreasing $\phi$ from the symmetric value of $\pi / 2$ gradually leads to regions of control. Further confirmation comes from the survival probability data shown in Fig. 6 for two points on each of the control landscapes with $\phi \neq 0$. It is intriguing to see that the wall of no control seen in Fig. [6A for $\lambda_{1} \approx 9.2$ is present for all the values of $\phi$ shown here. The reasons for such robustness is not clear at the moment. Note that such regions in Fig. [6 for $\phi \neq 0$ are characterized by large negative values of $C_{z}(\boldsymbol{\lambda})$. This seems to contradict the earlier works 39,40 which associated a large value for $\left|C_{z}\right|$ with extensive localization in phase space. However, as stated earlier, in the present case the interpretations are subtle with the sign of the chaotic level-velocity playing a key role. Our preliminary studies indicate that in such regions the assumption $\langle v\rangle_{r} \ll\left\langle v^{2}\right\rangle_{r}^{1 / 2}$ is weakened presumably due to an increased fraction of chaotic states. Further work is needed to understand this aspect. Nevertheless, the numerical results presented in Fig. [6indicate that regions of no control are signalled by $C_{z} \leq 0$ whereas $C_{z}>0$ implies control.

\section{CONCLUSIONS}

In summary, the strong field control landscapes in Fig. [6] show that nearly perfect control is obtained as long as there is no involvement of chaotic states in the dynamics. However, involvement of the chaotic states leads to lack of control and such regions on the control landscape are interspersed among controllable regions in a complicated fashion giving rise to a highly convoluted landscape topology. Some of these regions are particularly robust under field amplitude and relative phase variations (cf. Fig. 6). Given that convoluted landscapes are expected in OCT studies ${ }^{30,32}$ on classically chaotic systems, it is reasonable to expect that chaotic states can be associated with certain suboptimal features on quantum control landscapes. For systems with effective $\hbar \ll 1$ one expects ${ }^{38}$ several such plateau regions and the effectiveness of the control would depend sensitively on the initial state and field parameters.

We emphasize that the association of uncontrollable regions on the landscape with influence of the chaotic Floquet states is made possible solely due to the highly sensitive nature of the intensity-velocity correlator $C_{z}(\boldsymbol{\lambda})$ (cf. Eq. 8). The sensitivity of $C_{z}(\boldsymbol{\lambda})$ to the underlying phase space structures is evident from Fig. 5 and makes it an ideal candidate to understand the origins of suboptimal structures on the control landscape from a classicalquantum correspondence perspective. Moreover, $C_{z}(\boldsymbol{\lambda})$ can be obtained with a few Floquet calculations or via the parametric equations of motion ${ }^{8,41}$ and hence applicable to multidimensional driven systems. A further attractive feature, from the point of multidimensional systems, is that the correlator is capable of establishing the influence of the chaotic states on the control landscape without the need for visualizing/classifying the states in the underlying phase space.

Although the present work deals explicitly with a tunneling system, many of our observations are valid in other contexts as well. In general, controlling the decay (dissociation, ionization) of a state is expected to be complicated if one or several chaotic states participate in the dynamics. The complications will be reflected in the form of the optimal fields. This does not contradict the recent work ${ }^{22}$ by Takami and Fujisaki since they are concerned with controlling the transition between two random states in a completely chaotic system. It is the mixed regular-chaotic phase space scenario, a generic one for molecular systems, which is expected to exhibit some of the features seen in the current study. Finally, it would be interesting to see if the influence of chaotic states are indeed reflected in the topology of the quantum control level sets in an OCT calculation. In this regard the present work suggests that the intensityvelocity correlator could prove to be a useful tool. We are currently exploring the usefulness of the correlator in other systems and as a tool in formulating local control strategies. 


\section{Acknowledgments}

SK gratefully acknowledges useful discussions with Prof. Harshawardhan Wanare. It is a pleasure to thank Dr. Raj Chakrabarti for sharing his insights into control landscapes and for his crucial comments on a preprint version of the manuscript which led to the results in Eq. 11. Astha Sethi is funded by a Fellowship from the University Grants Commission, India.
${ }^{1}$ K. Bergmann, H. Theuer, and B. Shore, Rev. Mod. Phys. 70, 1003 (1998).

2 M. Shapiro and P. Brumer, Principles of the Quantum Control of Molecular Processes (Wiley, New York, 2003).

3 S. A. Rice and M. Zhao, Optical Control of Molecular Dynamics (Wiley, New York, 2000).

${ }^{4}$ H. A. Rabitz and W. Zhu, Acc. Chem. Res. 33, 572 (2000).

5 H. A. Rabitz, M. M. Hsieh, and C. M. Rosenthal, Science 303, 1998 (2004)

${ }^{6}$ R. Chakrabarti and H. A. Rabitz, Int. Rev. Phys. Chem. 26, 671 (2007).

7 Y. Dakhnovskii, R. Bavli, and H. Metiu, Phys. Rev. B 53, 4657 (1996).

8 A. K. Gupta, P. Gross, D. B. Bairagi, and M. K. Mishra, Chem. Phys. Lett. 257, 658 (1996).

9 M. E. Goggin and P. W. Milonni, Phys. Rev. A 38, 5174 (1988).

10 S. Gräfe, W. Kiefer and V. Engel, J. Chem. Phys. 127, 134306 (2007).

11 E. Wells, K. J. Betsch, C. W. S. Conover, M. J. DeWitt, D. Pinkham and R. R. Jones, Phys. Rev. A 72, 063406 (2005).

12 M. Wollenhaupt, A. Prakelt, C. Sarpe-Tudoran, D. Liese and T. Baumert, J. Mod. Opt. 52, 2187 (2005).

13 N. Sangouard, S. Guérin, M. Aminat-Talab, and H. R. Jauslin, Phys. Rev. Lett. 93, 223602 (2004).

14 F. L. Yip, D. A. Mazziotti, and H. A. Rabitz, J. Phys. Chem. A 107, 7264 (2003).

15 T. Cheng, H. Darmawan, and A. Brown, Phys. Rev. A 75, 013411 (2007); D. Sugny, C. Kontz, M. Ndong, Y. Justum, G. Dive, and M. Desouter-Lecomte, Phys. Rev. A 74, 043419 (2006).

16 S. Kohler, R. Utermann, P. Hänggi, and T. Dittrich, Phys. Rev. E 58, 7219 (1998).

17 M. Latka, P. Grigolini and B. J. West, Phys. Rev. E 50, R3299 (1994).

18 J. Gong and P. Brumer, Annu. Rev. Phys. Chem. 56, 1 (2005).

19 K. Na and L. E. Reichl, Phys. Rev. A 72, 013402 (2005); Chaos, Solitons and Fractals, 25, 185 (2005).

20 J. Roslund, M. Roth, and H. Rabitz, Phys. Rev. A 74, 043414 (2006).

21 A. F. Bartelt, T. Feurer, and L. Wöste, Chem. Phys. 318, 207 (2005).

${ }^{22}$ T. Takami and H. Fujisaki, Phys. Rev. E 75, 036219 (2007).

23 M. Grifoni and P. Hänggi, Phys. Rep. 304, 229 (1998).

${ }^{24}$ F. Grossmann, T. Dittrich, P. Jung, and P. Hänggi, Phys. Rev. Lett. 67, 516 (1991).

25 M. Holthaus, Phys. Rev. Lett. 69, 1596 (1992); R. Bavli and H. Metiu, Phys. Rev. Lett. 69, 1986 (1992); S. Ya. Kilin, P. R. Berman, and T. M. Maevskaya, Phys. Rev. Lett. 76, 3297 (1996); J. Y. Shin, Phys. Rev. E 54, 289
(1996); A. Igarashi and H. Yamada, Chem. Phys. 327, 395 (2006).

26 D. Farrelly and J. A. Milligan, Phys. Rev. E 47, R2225 (1993).

27 W. A. Lin and L. E. Ballentine, Phys. Rev. Lett. 65, 2927 (1990)

28 M. Ndong, D. Lauvergnat, X. Chapuisat, and M. DesouterLecomte, J. Chem. Phys. 126, 244505 (2007); U. Troppmann, C. Gollub, and R. de Vivie-Riedle, New J. Phys. 8, 100 (2006); S. Suzuki, K. Mishima, and K. Yamashita, Chem. Phys. Lett. 410, 358 (2005).

29 A. F. Terzis, S. Kosionis, and E. Paspalakis, J. Phys. B: At. Mol. Opt. Phys. 40, S331 (2007).

30 C. D. Schwieters and H. Rabitz, Phys. Rev. A 48, 2549 (1993).

31 F. Ehlotzky, Phys. Rep. 345, 175 (2001); S. Huang, C. Chandre, and T. Uzer, J. Phys. B: At. Mol. Opt. Phys. 40, F181 (2007); L. Sirko and P. M. Koch, Phys. Rev. Lett. 89, 274101 (2002); S. Denisov, L. Morales-Molina, S. Flach, and P. Hänggi, Phys. Rev. A 75, 063424 (2007); V. Constantoudis and C. A. Nicolaides, J. Chem. Phys. 122, 084118 (2005); I. Vrábel and W. Jakubetz, J. Chem. Phys. 118, 7366 (2003); V. S. Batista and P. Brumer, Phys. Rev. Lett. 89, 143201 (2002); H. Ohmura and M. Tachiya, Phys. Rev. A 77, 023408 (2008); R. Tehini and D. Sugny, Phys. Rev. A 77, 023407 (2008).

32 C. D. Schwieters and H. Rabitz, Phys. Rev. A 44, 5224 (1991).

33 J. Plata and J. M. G. Llorente, J. Phys. A: Math. Gen. 25, L303 (1992).

34 J. H. Shirley, Phys. Rev. 138, B979 (1965).

35 H. Sambe, Phys. Rev. A 7, 2203 (1973).

36 C. Eltschka and P. Schlagheck, Phys. Rev. Lett. 94, 014101 (2005); S. Wimberger, P. Schlagheck, C. Eltschka, and A. Buchleitner, Phys. Rev. Lett. 97, 043001 (2006); A. Mouchet, C. Eltschka, and P. Schlagheck, Phys. Rev. E 74, 026211 (2006).

37 S. Keshavamurthy, Phys. Rev. E 72, 045203(R) (2005); S. Keshavamurthy, J. Chem. Phys. 122, 114109 (2005); S. Keshavamurthy, Int. Rev. Phys. Chem. 26, 521 (2007).

38 S. Tomsovic and D. Ullmo, Phys. Rev. E 50, 145 (1994).

39 S. Tomsovic, Phys. Rev. Lett. 77, 4158 (1996).

40 N. R. Cerruti, S. Keshavamurthy, and S. Tomsovic, Phys. Rev. E 68, 056205 (2003); S. Keshavamurthy, N. R. Cerruti and S. Tomsovic, J. Chem. Phys. 117, 4168 (2002); A. Lakshminarayan, N. R. Cerruti, and S. Tomsovic, Phys. Rev. E 63, 016209 (2000).

41 D. A. Mazziotti, M. K. Mishra, and H. A. Rabitz, J. Phys. Chem. 99, 112 (1995); D. A. Mazziotti, Senior thesis (Department of Chemistry, Princeton University, 1995); D. A. Mazziotti and H. A. Rabitz, Mol. Phys. 89, 171 (1996). 